\title{
Association Between Empathy Competence and Emotional Intelligence Among Medical Undergraduates: A Cross-sectional Study
}

\author{
Lou Pengyu, Hu Jinwei* \\ Shandong", Weifang Medical University, Weifang, China \\ Email address: \\ Loupy@wfmc.edu.cn (Lou Pengyu),Hujw99@163.com (Hu Jinwei) \\ ${ }^{*}$ Corresponding author
}

College of Public Health and Management, Collaborative Innovation Center for Major Social Risk Prediction and Governance of "Healthy

\section{To cite this article:}

Lou Pengyu, Hu Jinwei. Association Between Empathy Competence and Emotional Intelligence Among Medical Undergraduates: A Cross-sectional Study. American Journal of Applied Psychology. Vol. 8, No. 3, 2019, pp. 57-63. doi: 10.11648/j.ajap.20190803.11

Received: April 1, 2019; Accepted: June 5, 2019; Published: June 27, 2019

\begin{abstract}
Purpose Study on the relationship between empathy competence and emotional intelligence used to provide suggestions for alleviating miscommunication that leads to misunderstandings between doctors and patients. Methods Stratified random cluster sampling was employed for questionnaires from 688 medical undergraduates in Weifang medical university (a provincial colleges and universities in Weifang City, Shandong Province, China) by means of PES and EIS. Results There were significant differences in self-regulation and emotions regulation of others between genders $(P<0.05)$, and there were significant differences in emotional sharing between genders $(P<0.05)$. There was no significant difference in emotional intelligence and empathy competence among domicile $(P>0.05)$. There were significant differences in all dimensions of emotional intelligence except self-regulated emotion between grades $(P<0.05)$, and there were significant differences in emotional sharing, positive care and empathy competence among grades $(P<0.05)$. Medical undergraduates' emotional intelligence is positively correlated with empathy competence. Conclusion Medical colleges and universities should establish a systematic, professional and organic curriculum system of humanities and social sciences, and attach importance to the continuity of emotional intelligence and empathy training.
\end{abstract}

Keywords: Empathy Competence, Emotional Intelligence, Doctor-patient Relationship, Medical Undergraduates, Medical Humanities Education

\section{Introduction}

Empathy is a complex concept. It is mainly embodied in the ability to understand others, which allows others to feel accepted and cared for by the empathy [1]. Through empathy, we can adjust cognitive bias in communication, promote emotional resonance, ensure the effectiveness of information exchange, and help to build good interpersonal relationships. Nowadays, the term "empathy" is more common in the medical and health fields. As a subjective, multidimensional and dynamic indicator for evaluating the degree of individual socialization [2]. Empathy plays an increasingly important role in clinical trials, study on humanistic quality training and medical scene of medical staff [3-5]. In clinical trials, empathy nursing can improve patients' bad mood and improve their quality of life effectively [6]. In terms of medical humanistic quality, empathy ability was positively correlated with nurse-patient communication ability, self-efficacy, professional attitude, and affected nursing job satisfaction [7]. At the same time, empathy training is conducive to improving cultural accomplishment.

Empathy also plays an important role in medical scenarios. When medical workers can experience patients' pain, it will be beneficial to the harmony of treatment effect and doctor-patient relationship [8]. The doctor-patient relationship is very important for the successful diagnosis and treatment of patients [9]. Contradictions and misunderstandings between doctors and patients in China have intensified year by year. Not only have patients been dissatisfied with the process of diagnosis and treatment, but their physical and mental 
suffering has been aggravated as well. Doctors were also deeply disturbed by misunderstandings between doctors and patients which affected their work [10]. Some studies have shown that empathy was a key factor for doctors to implement diagnosis and treatment, which had become an important factor affecting the quality of medical staff service and the establishment of a harmonious doctor-patient atmosphere in the environment of tense doctor-patient relationship [11]. Empathy can help the diagnosis of disease, produce therapeutic effects, such as creating a positive impact on patients' satisfaction, compliance and the reduction of medical expenditure, and reduction in the doctor's work pressure and enhancement of their professional identity $[12,13]$.

Emotional intelligence refers to the ability of individuals to perceive, understand and utilize the emotions of themselves and others, and to use these information as a guide for their own thinking and action [14]. Studies have shown that emotional intelligence of medical postgraduates is associated with empathy, and good control of their own emotions has a positive impact on good relationships with their superiors and colleagues [15]. In clinical work, nursing students and nurses are equally applicable. Emotional intelligence is positively correlated with empathy. Nurses with high emotional intelligence can produce beneficial behaviors to patients through emotional regulation, which can be more recognized by patients and their families [16].

At present, there are few studies on relationship between emotional intelligence and empathy involving medical postgraduates and nurses. According to the academic standards stipulated in the Higher Education Law of the People's Republic of China, there are differences in the educational methods between medical postgraduates and undergraduates. Postgraduate education has a deeper understanding of medical humanities quality because it is closer to clinical teaching [17]. In terms of emotions, medical postgraduates are more anxious and nervous than undergraduates, showing melancholy, worry and nervousness [18]. Because there are many differences between medical and nursing science, medical postgraduates and undergraduates, and undergraduate education is an important stage to master basic theory, basic knowledge, basic skills and methods, it is necessary to study the relationship between emotional intelligence and empathy of medical undergraduates. In this paper, the characteristics of emotional intelligence and empathy competence of medical undergraduates were investigated, and the ways of affecting emotional intelligence and its dimensions on empathy competence were explored, so as to provide basis for improving the mental health status and empathy competence of medical undergraduates.

\section{Sample and Methods}

\subsection{Sample}

The cross-sectional study was conducted in Weifang medical university (a provincial colleges and universities in Weifang City, Shandong Province, China), carrying about
4000 medical undergraduates $(30.77 \%$ of total university population) from May 2018 to July 2018. We conducted a multistage, stratified, and cluster sampling scheme that used the class as a sampling unit, stratified by the type of grade, and randomly selected at the class level. We randomly selected 3 classes from each grade, and all 700 potential participants from 15 classes were included in the study. The data were collected in the classroom environment by a pen and paper self-report questionnaire. Trained research assistants provided the brief introduction and notes about the questionnaire, the questionnaire was completed under quiet classroom conditions within the given 10-min period. The inclusion criteria for the participants were as follows: (1) consent to participate in the study; (2) In the classroom on the day of the data collection. In the end, we screened 688 eligible participants from 700 potential participants, for a response rate of $98.3 \%$.

\subsection{Methods}

Physician Empathy Scale.

The Physician Empathy Scale is a measurement tool for Chinese physicians specially [19]. It fully incorporates patients' understanding and needs of physicians' empathy behavior, which is different from the J Jefferson scale of physician empathy. It consists of 18 items and the following four dimensions: perspective-taking ability (5 items), emotional sharing ability (4 items), positive care ability (4 items), and empathy expression ability (5 items). Responses are categorized into a five-point Likert scale that evaluates the frequency $(1$ =Very disapproval; 2 = Disapproval; $3=$ Common; 4= Approval; 5 =Very approval). Higher scores indicate better empathy. The Cronbach's alpha coefficient was satisfactory at 0.840 .

Emotional Intelligence Scale.

The Emotional Intelligence Scale is compiled by Schutte et al. [20]. The Chinese version is introduced by Wang Caikang [21]. It had good reliability and validity in college students [22]. A total of 33 items and the following four dimensions: Emotional perception (12 items), Self-regulation (6 items), regulation of others (10 items), Emotional use (5 items). Responses are categorized into a five-point Likert scale that evaluates the frequency $(1=$ Very inconsistent; $2=$ Inconsistent; 3 = Unclear; 4 =Consistent; 5 =Very consistent), Higher scores indicate better emotional Intelligence, the Cronbach's alpha coefficient was satisfactory at 0.83 .

Socio-demographic characteristics.

A socio-demographic information sheet covered basic participant information, including gender, domicile (Domicile refers to the family address, which is divided into urban and rural areas.), grade (In China, clinical medicine undergraduate specialty adopts a five-year teaching mode. The educational management functions of students in grades 1, 2 and 3 are assigned to basic medical colleges. Affiliated hospitals undertake the teaching tasks of full-time students in grades 4 and 5 in education management and internship.). 


\subsection{Statistical Analysis}

EpiData 2.0 (EpiData Association, Odense, Denmark) was used to establish the database SPSS 18 for Windows (SPSS, Inc., Chicago, IL) was used for statistical processing of data. The scores of each dimension were described by $\bar{x} \pm s$, and the data were analyzed by independent sample t test, analysis of variance and correlation analysis. A $p$ value of less than 0.05 was accepted as statistically significant.

\section{Results}

\subsection{Empathy Competence and Emotional Intelligence of Medical Undergraduates}

The mean of all subjects on empathy competence scored $4.03 \pm 0.63$. Gender differences in emotional sharing were statistically significant $(t=2.683, P<0.05)$. There was no significant difference among domicile. There were significant differences in empathy competence of grades except perspective-taking and empathy expression $(F=1.310 \sim 1.985$,
$P>0.05)$. The mean of all subjects on emotional intelligence scored $3.78 \pm 0.51$. There were statistically significant differences in gender between self-regulation and regulation of others $(t=2.206 \sim 2.967, P<0.05)$. There were no significant differences between domicile $(t=0.794 \sim 1.315, P>$ 0.05). There were significant differences in emotional intelligence of grades except self-regulation and emotional use $(F=1.345, P>0.05)$ (Table 1 and Table 2$)$. Grades in the ANOVA analysis of empathy competence and emotional intelligence, $F$ value reached a significant level. In order to further clarify which paired groups have significant differences in the mean of dependent variables, it is necessary to carry out post hoc analysis on the mean differences across the five educational levels (by honestly significant difference). As far as empathy competence is concerned, the second-grade group is significantly higher than the third-grade group, and the second-grade group is significantly higher than the fourth-grade group. As far as emotional intelligence is concerned, the fourth-grade group is significantly higher than the first-grade group, and the fifth-grade group is significantly higher than the first-grade group (Table 3 ).

Table 1. Comparison of empathy competence scores among different groups.

\begin{tabular}{|c|c|c|c|c|c|c|c|c|}
\hline Variables & Category & Num & $t / F$ & Perspective-taking & $\begin{array}{l}\text { Emotional } \\
\text { Sharing } \\
\end{array}$ & $\begin{array}{l}\text { Positive } \\
\text { Care } \\
\end{array}$ & $\begin{array}{l}\text { Empathy } \\
\text { expression }\end{array}$ & $\begin{array}{l}\text { Empathy } \\
\text { competence }\end{array}$ \\
\hline \multirow[t]{4}{*}{ Gender } & male & 358 & & $4.04 \pm 0.68$ & $3.95 \pm 0.82$ & $4.01 \pm 0.67$ & $4.222 \pm 1.06$ & $4.03 \pm 0.65$ \\
\hline & female & 330 & & $4.10 \pm 0.66$ & $3.81 \pm 0.77$ & $4.00 \pm 0.68$ & $4.28 \pm 0.90$ & $4.04 \pm 0.62$ \\
\hline & & & $t$ & -1.197 & $2.683 *$ & 0.215 & -0.648 & -0.255 \\
\hline & & & $P$ & 0.112 & 0.014 & 0.473 & 0.223 & 0.143 \\
\hline \multirow[t]{4}{*}{ Domicile } & urban & 340 & & $4.09 \pm 0.63$ & $3.82 \pm 0.80$ & $4.01 \pm 0.67$ & $4.26 \pm 1.03$ & $4.04 \pm 0.63$ \\
\hline & rural & 348 & & $4.04 \pm 0.71$ & $3.85 \pm 0.79$ & $4.00 \pm 0.68$ & $4.23 \pm 0.93$ & $4.03 \pm 0.64$ \\
\hline & & & $t$ & 0.800 & -0.504 & 1.732 & 0.386 & 0.126 \\
\hline & & & $P$ & 0.405 & 0.306 & 0.360 & 0.727 & 0.303 \\
\hline \multirow[t]{7}{*}{ Grades } & freshmen & 140 & & $4.04 \pm 0.68$ & $3.93 \pm 0.85$ & $4.03 \pm 0.67$ & $4.23 \pm 0.74$ & $4.06 \pm 0.61$ \\
\hline & sophomores & 148 & & $4.16 \pm 0.71$ & $4.18 \pm 0.75$ & $4.20 \pm 0.72$ & $4.36 \pm 1.19$ & $4.22 \pm 0.73$ \\
\hline & juniors & 128 & & $3.96 \pm 0.77$ & $3.74 \pm 0.81$ & $3.93 \pm 0.72$ & $4.17 \pm 1.53$ & $3.95 \pm 0.78$ \\
\hline & seniors & 140 & & $4.03 \pm 0.54$ & $3.61 \pm 0.70$ & $3.84 \pm 0.57$ & $4.14 \pm 0.54$ & $3.91 \pm 0.47$ \\
\hline & fifth-year students & 132 & & $4.13 \pm 0.63$ & $3.64 \pm 0.71$ & $4.00 \pm 0.61$ & $4.34 \pm 9.52$ & $4.02 \pm 0.47$ \\
\hline & & & $F$ & 1.985 & $13.242 *$ & $5.874 *$ & 1.310 & $5.131 *$ \\
\hline & & & $P$ & 0.095 & 0.000 & 0.000 & 0.256 & 0.000 \\
\hline
\end{tabular}

Note: *. Significant correlation at 0.05 level (bilateral).

Table 2. Comparison of emotional intelligence scores among different groups.

\begin{tabular}{|c|c|c|c|c|c|c|c|c|}
\hline Variables & Category & Num & $t / F$ & $\begin{array}{l}\text { Emotional } \\
\text { Perception }\end{array}$ & Self-regulation & $\begin{array}{l}\text { Others } \\
\text { regulation }\end{array}$ & $\begin{array}{l}\text { Emotional } \\
\text { Use }\end{array}$ & $\begin{array}{l}\text { Emotional } \\
\text { Intelligence }\end{array}$ \\
\hline \multirow[t]{4}{*}{ Gender } & male & 358 & & $3.66 \pm 0.56$ & $3.71 \pm 0.48$ & $3.70 \pm 0.59$ & $3.89 \pm 0.58$ & $3.79 \pm 0.49$ \\
\hline & female & 330 & & $3.62 \pm 0.56$ & $3.89 \pm 0.55$ & $3.91 \pm 0.62$ & $3.88 \pm 0.60$ & $3.78 \pm 0.53$ \\
\hline & & & $t$ & 0.760 & $-2.206^{*}$ & $-2.967 *$ & 0.080 & 0.187 \\
\hline & & & $P$ & 0.148 & 0.015 & 0.004 & 0.379 & 0.618 \\
\hline \multirow[t]{4}{*}{ Domicile } & urban & 340 & & $3.62 \pm 0.48$ & $3.74 \pm 0.52$ & $3.94 \pm 0.61$ & $3.90 \pm 0.56$ & $3.80 \pm 0.49$ \\
\hline & rural & 348 & & $3.66 \pm 0.63$ & $3.70 \pm 0.51$ & $3.88 \pm 0.61$ & $3.87 \pm 0.61$ & $3.78 \pm 0.53$ \\
\hline & & & $t$ & -0.794 & 0.949 & 1.315 & 0.587 & 0.454 \\
\hline & & & $P$ & 0.580 & 0.209 & 0.396 & 0.917 & 0.379 \\
\hline \multirow[t]{6}{*}{ Grades } & freshmen & 140 & & $3.52 \pm 0.34$ & $3.77 \pm 0.46$ & $3.70 \pm 0.62$ & $3.86 \pm 0.51$ & $3.61 \pm 0.51$ \\
\hline & sophomores & 148 & & $3.57 \pm 0.80$ & $3.73 \pm 0.59$ & $3.81 \pm 0.73$ & $3.93 \pm 0.68$ & $3.72 \pm 0.52$ \\
\hline & juniors & 128 & & $3.62 \pm 0.41$ & $3.62 \pm 0.52$ & $3.84 \pm 0.61$ & $3.88 \pm 0.64$ & $3.73 \pm 0.48$ \\
\hline & seniors & 140 & & $3.66 \pm 0.58$ & $3.68 \pm 0.41$ & $3.95 \pm 0.55$ & $3.80 \pm 0.49$ & $3.86 \pm 0.57$ \\
\hline & fifth-year students & 132 & & $3.75 \pm 0.55$ & $3.77 \pm 0.53$ & $4.00 \pm 0.46$ & $3.94 \pm 0.59$ & $3.89 \pm 0.41$ \\
\hline & & & $P$ & 0.027 & 0.075 & 0.039 & 0.252 & 0.006 \\
\hline
\end{tabular}

Note: *. Significant correlation at 0.05 level (bilateral). 
Table 3. Summary table of variance analysis of differences in emotional intelligence and empathy competence of different grades.

\begin{tabular}{|c|c|c|c|c|c|c|}
\hline & & Sum of Squares & df & Mean Square & $\mathbf{F}$ & HSD \\
\hline \multirow{3}{*}{ Empathy competence } & Between Groups & 8.491 & 4 & 2.123 & $5.359^{*}$ & second-grade $>$ third-grade \\
\hline & Within Groups & 270.558 & 683 & .396 & & second-grade $>$ fourth-grade \\
\hline & Total & 279.049 & 687 & & & \\
\hline \multirow[t]{2}{*}{ Emotional intelligence } & Within Groups & 177.204 & 683 & .259 & & fifth-grade $>$ first-grade \\
\hline & Total & 180.398 & 687 & & & \\
\hline
\end{tabular}

Note: *. Significant correlation at 0.05 level (bilateral).

\subsection{Correlation Analysis Between Medical Undergraduates' Emotional Intelligence and Empathy Competence}

There was a significant positive correlation between emotional intelligence and empathy competence $(P<0.01)$, and the correlation coefficient was 0.609 (Table 4).

Table 4. Correlation coefficient between emotional intelligence and empathy competence of medical undergraduates $(r, n=688)$.

\begin{tabular}{|c|c|c|c|c|c|}
\hline Variables & Perspective-taking & Emotional Sharing & Positive Care & Empathy expression & Empathy competence \\
\hline Emotional Perception & $0.481^{* *}$ & $0.369^{* *}$ & $0.505^{* *}$ & $0.343^{* *}$ & $0.506^{* *}$ \\
\hline Self-regulation & $0.525^{* *}$ & $0.421^{* *}$ & $0.506^{* *}$ & $0.412^{* *}$ & $0.564^{* *}$ \\
\hline Regulation of others & $0.511^{* *}$ & $0.447^{* *}$ & $0.532^{* *}$ & $0.409^{* *}$ & $0.572^{* *}$ \\
\hline Emotional Use & $0.506^{* *}$ & $0.420^{* *}$ & $0.503^{* *}$ & $0.380^{* *}$ & $0.544^{* *}$ \\
\hline Emotional Intelligence & $0.565^{* *}$ & $0.462^{* *}$ & $0.569^{* *}$ & $0.429^{* *}$ & $0.609^{* *}$ \\
\hline
\end{tabular}

\subsection{Regression Analysis of Medical Undergraduates' Emotional Intelligence on Empathy Competence}

Using emotional intelligence as a predictor and empathy competence as dependent variable, linear regression analysis was conducted. Before regression analysis was done, scatter plots were used to test whether there was linear relationship between the two variables. Figure 1 provides a graph showing that the linear relationship between the two variables of emotional intelligence and empathy competence was obvious, which was suitable for regression analysis.

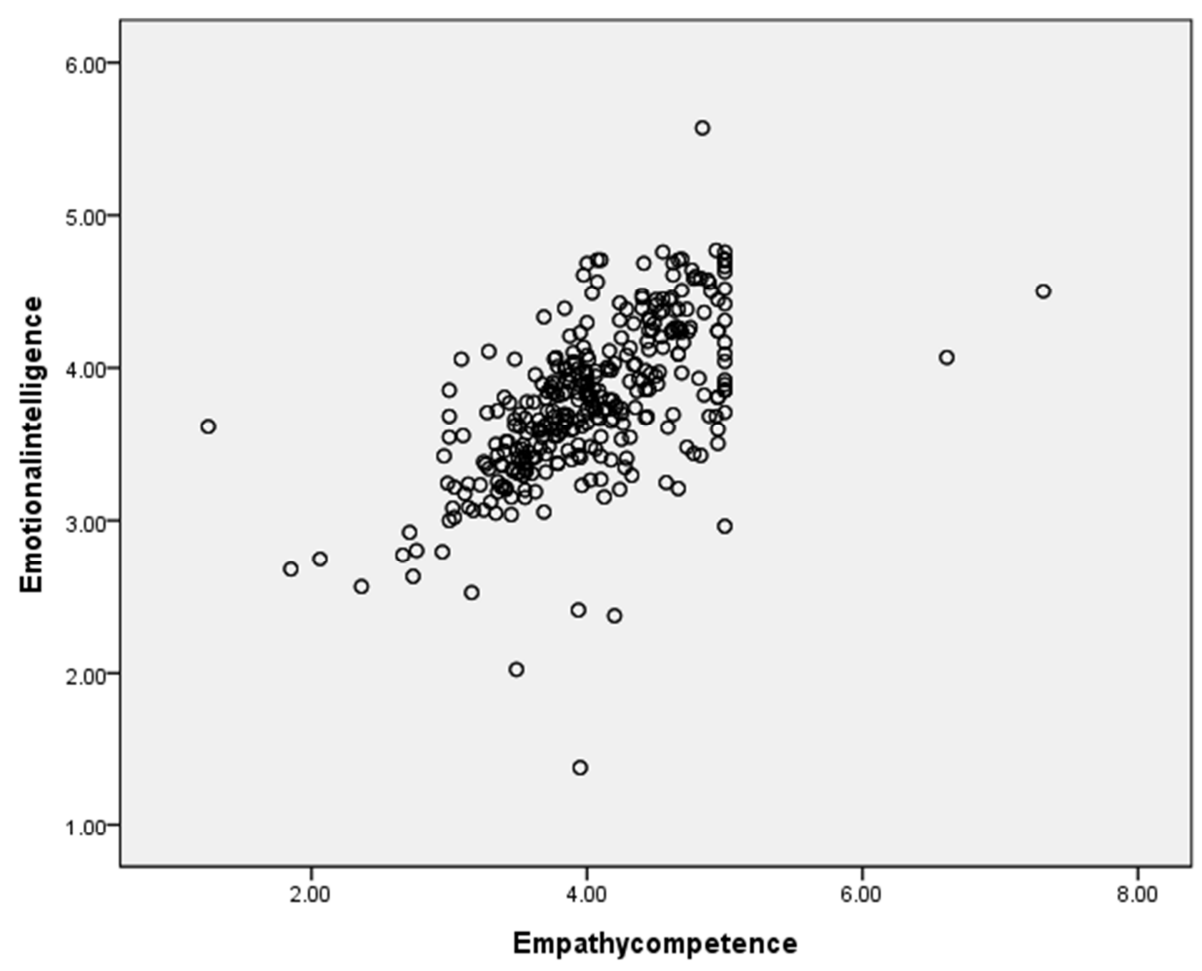

Figure 1. Scatter diagram of emotional intelligence and empathy competence.

The predictive effects of emotional intelligence on empathy competence and various dimensions were analyzed by linear regression method (Table 5). The results showed that emotional intelligence of medical undergraduates had a good predictive power on empathy competence, explaining $47.1 \%$ of the variation of empathy competence $(F(1,688)=400.486, P<$ $0.01)$. The fitting result of emotional intelligence on empathy competence was $y=1.136+0.766 x$. In order to test the 
effectiveness of emotional intelligence on the regression model of empathy competence, residual analysis was used to test the fitting results, as shown in Figure 2. Most of the scatter plots were scattered near the slant line, indicating that the residual was normal distributed, so the regression model of emotional intelligence on empathy competence was established.

Table 5. Regression analysis of medical undergraduates' emotional intelligence on empathy competence。

\begin{tabular}{lllllll}
\hline & $\boldsymbol{R}^{2}$ & Adjusted $\boldsymbol{R}^{2}$ & $\boldsymbol{F}$ & $\boldsymbol{B}$ & Sig. \\
\hline Empathy competence & 0.471 & 0.470 & 400.486 & 0.766 & 0.000 \\
Perspective-taking & 0.320 & 0.319 & 320.610 & 0.755 & 17.906 \\
Emotional sharing & 0.214 & 0.213 & 184.369 & 0.726 & 13.578 \\
Positive care & 0.324 & 0.323 & 327.083 & 0.757 & 0.000 \\
Empathy expression & 0.184 & 0.183 & 154.200 & 0.836 & 0.000 \\
\hline
\end{tabular}

Normal P-P Plot of Regression Standardized Residual

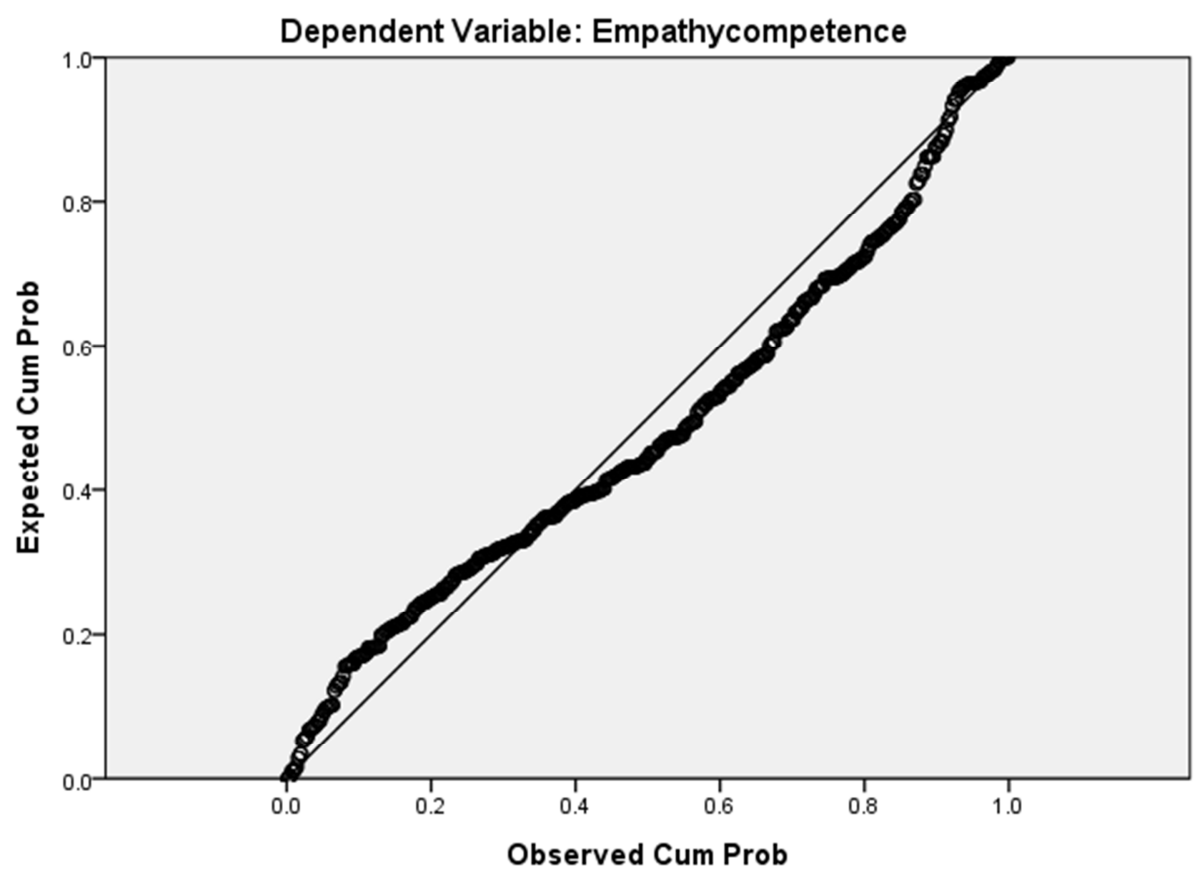

Figure 2. Normalized residuals normal P-P diagram.

\section{Discussion}

The results showed that there were significant differences between females and males in self-regulation and emotions regulation of others, which was consistent with the results of Xiao Jing [23]. Female's ability to regulate their emotions is stronger because of gender characteristics and social roles. Females were more gentle and modest when communicating with others, more encouraged to express and care for others, and more acceptable. There was no difference in emotional intelligence and other dimensions between students' domicile which was consistent with the results of Zhao Weiyan [24]. Emotional intelligence can be improved by learning. Psychological courses such as mental health education, health education and social psychology for college students make no significant difference in the emotional intelligence on medical undergraduates in different domicile. The effect of grade on emotional intelligence was consistent with the results of Sun Lihong [25]. Emotional intelligence increased with the growth of grade, which was related to the clear goal of development, the improvement of career planning ability, and rich medical knowledge.

The results showed that there were significant differences in emotional sharing scores between genders, which might be related to gender and personality differences. Females were more willing to share their stories and feelings in the process of interpersonal communication, and understand other peoples' emotions more easily. This study did not find the influence of students' domicile on empathy competence, which was consistent with the results of Yu Wanwan [26]. Empathy is not only a personality trait, but also a state of ability, which is acquired. It could be continuously improved through acquired learning and practice [27]. Empathy competence decreased with the increase of grade, which was consistent with the results of Zhai Chunxiao [28]. The second-grade group is significantly higher than the third-grade group, and the second-grade group is significantly higher than the fourth-grade group. This was related to the humanities curriculum for clinical undergraduates. The compulsory courses of humanities in the first-grade are mainly in ideological education and public basic education. The focus of humanities in the second-grade is mainly medical ethics and medical psychology. Medical 
undergraduates who are interested in humanities can also choose medical history, health law, social psychology, social medicine, health economics, health service research, eloquence art, etiquette and self-cultivation. These two stages of rich humanities courses enhance the empathy ability of medical undergraduates. The main courses offered in the third and fourth grades are basic medicine and clinical medicine. There are many courses and heavy learning tasks. The humanities courses such as law and medical service marketing, which can be optional at this stage, are difficult to further enhance the ability of empathy. This may be the reason that the ability of empathy in the third and fourth grades is obviously reduced. Empathy competence will be affected by lack of coherence in the cultivation of humanistic competence.

There was a significant positive correlation between emotional intelligence and empathy competence. Improving emotional intelligence of medical undergraduates could enhance their empathy competences, which was consistent with the results of Wang Shengfeng [29]. Medical undergraduates of high emotional intelligence could better regulate their negative emotions, correctly understand and feel others emotions, better communicate, and better interpersonal relationships with others. In terms of specific dimensions, emotional perception was positively correlated with five dimensions of empathy competence. Emotional perception was the ability to identify emotions from own physical and emotional state, and from others speech, voice, expression and behavior. Medical undergraduates with high emotional perception were more likely to identify others negative emotions during the listening process, used empathy to regulate emotions, and adopt appropriate expressions to eliminate negative emotions more quickly, and gained the trust of others. Self-regulation and regulation of others were positively correlated with five dimensions of empathy competence. Medical undergraduates with high emotional intelligence could actively regulate their own and others emotions, maintain own good physical and mental state, who were more willing to communicate and reach agreement with others, maintain harmonious interpersonal relationship with others, had strong social adaptability, and were more likely to succeed in their study, life and work. It was particularly important for clear motivation, creative thinking, flexible planning of emotional use in difficult situation communication, and could take to create a harmonious atmosphere, positive emotions, correct and effective action to improve the situation.

\section{Conclusion}

In a word, emotional intelligence of medical undergraduates needed to be improved, and emotional intelligence was positively related to empathy competence. Therefore, in addition to imparting professional knowledge and skills, medical colleges and universities should establish a systematic, professional and organic curriculum system of humanities and social sciences. They should emphasize the cultivation of emotional intelligence, improve the ability of medical undergraduates to understand, and express their own feelings and experience of others' emotions. Additionally, they should actively guide medical undergraduates to correctly understand the difficulties and environment in their study and life, learn to use appropriate and positive ways to deal with cooperation and competition, and improve psychological endurance. By actively guiding medical undergraduates to participate in school activities and social practice, they will cultivate the ability to emotions regulation of others and emotional use, and promote the improvement of empathy competence.

\section{Acknowledgements}

This research was financially supported by Planning Fund Youth Fund Project of Ministry of Education Humanities and Social Sciences Research (Grant NO. 15YJCZH065) and Project of Scientific Research Development Plan of Shandong Higher Education Institutions (Grant NO. J18RB045).

\section{References}

[1] Duan CM, Hill CE. The current State of empathy research [J]. J Couns Psychol, 1996, 43 (3): 261-274.

[2] McMillan LR, Shannon D. Program evaluation of nursing school instruction in measuring students' perceived competence to empathetically communicate with patients $[\mathrm{J}]$. Nurs Educ Perspect, 2011, 32 (3): 150-4.

[3] Chrisman-Khawam L, Abdullah N, Dhoopar A. Teaching health-care trainees empathy and homelessness IQ through service learning, reflective practice, and altruistic attribution [J]. Int J Psychiatry Med, 2017, 52 (3): 245-254.

[4] La Monica El, Wolf R M, Madea A R, et al. Empathy and nursing care outcomes [J]. Sch Inq Nurs Pract, 1987, 1 (3): 197-213.

[5] Wang Juan, Li Li Li, Lin Wenjuan, et al. Empathy - a new vision for improving doctor-patient communication [J]. Medicine and Philosophy (Humanities and Social Medicine Edition), 2011, 32 (11): 25-27.

[6] Leonard CR, Zomorodi M, Foster BB. The impact of caring: Teaching students empathy through the patient voice $[\mathrm{J}]$. Creat Nurs. 2018, 24 (1): 62-66.

[7] Wei Hua, Huang Jingtang, Shen Peihong. Study on the relationship between empathic fatigue and occupational stress and burnout among pre-hospital emergency nurses [J]. China Occupational Medicine, 2016, 43 (2): 155-160.

[8] Zhang Ying, Mei SongLi, Xu Jun, et al. Cope with the doctor-patient relationship in predicament by psychological empathy theory [J]. Medicine and Philosophy, 2014, 35 (10): $51-53$.

[9] Yuguero O, Ramon M L, Esquerda M, et al. Association between low empathy and high burnout among primary care physicians and nurses in Lleida, Spain [J]. European Journal of General Practice, 2017, 23 (1): 4-10. 
[10] Liu Hanlong, Liu Huijun. Empathy: An Ethical Practical Approach to Reconstructing Trust Relations between Doctors and Patients [J]. Chinese Medical Ethics, 2012, 25 (3): 347-349.

[11] Hojat M, Mangione S, Nasca T J, et al. The Jefferson Scale of physical empathy: development and preliminary psychometric data [J]. Educ Psychol Meas, 2001, 61 (2): 349-365.

[12] LIU Hanlong, Liu Huijun. Empathy: An Ethical Practical Approach to Reconstructing Trust Relations between Doctors and Patients [J]. Chinese Medical Ethics, 2012, 25 (3): 347-349. (In Chinese).

[13] Wagner, P. J. \& Moseley, G. C. Physicians emotional intelligence and patient satisfaction. Family Medicine, 2002, 34 (10): 750-754.

[14] SALOVER P, MAYER JD. Emotional intelligence [J]. Imag Cognperson, 1990, 9 (3): 185-211.

[15] Abhijeet F, Gurvinder K, Rajeev S, et al. Study of emotional intelligence and empathy in medical postgraduates [J]. Indian Journal of Psychiatry, 2011, 53 (2): 140-145.

[16] Mi Yan. A study on the relationship between empathy ability and emotional intelligence of nursing interns in higher vocational colleges [J]. Journal of Community Medicine, 2016, 14 (21): 26-28.

[17] Yuan Rongdi. Teaching experience of ophthalmology undergraduates and postgraduates [J]. Lab Med Clin, 2016, 13 (12): 1738-1739.

[18] Jia Yanbin, Ye Mingzhi, Xiao Plan. A comparative analysis of 16 personalities of medical postgraduates and undergraduates [J]. Journal of Jinan University (Medicine Edition), 2011, 22 (2): $84-88$.

[19] XU Min, Wang Yungui. The compilation of self-assessment questionnaire on physician empathy [J]. Journal of Third Military Medical University, 2017, 39 (12): 1309-1304.

[20] Schutte NS, MaLOUFFJM. Emotional intelligence and interpersonal relations [J]. JOC Psychol, 2001, 141 (4): 523-536.

[21] Wang Caikang. A Study on Emotional Intelligence of Juvenile Delinquents and Its Relevant Personality Factors [J]. Chinese Journal of Mental Health, 2002, 16 (8): 566-567.

[22] Xiao Rong, Zhang Xiaoyuan and Zhao Jiubo. The relationship between college students' sense of meaning in life and their mental health [J]. School Health in China, 2010, 31 (4): 445-446.

[23] XIAO Jing, Wang Fei, Ge Hua, et al. Medical students' learning burnout and emotional intelligence $[\mathrm{J}]$. Chinese School Health, 2013, 34 (12): 1442-1444.

[24] ZHAO Weiyan, Li Meng, Li Qingqing. The relationship between college students' emotional intelligence and career decision-making self-efficacy [J]. Chinese Journal of Health Psychology, 2015, 23 (8): 1178-1182.

[25] SUN Lihong. An analysis of emotional intelligence of undergraduate medical students in a medical college in Shandong province [J]. Health Vocational Education, 2017, 35 (16): 76-78.

[26] YU Wanwan, Wang Kai, Liang Zheng. Research on the relationship between empathy and parenting styles of medical college students [J]. School Health of China, 2012, 33 (1): 28-30.

[27] Kataoka H, Iwase T1, Ogawa $\mathrm{H}$ et al. Can communication skills training improve empathy? A six-year longitudinal study of medical students in Japan [J]. MedTeach, 2018, 22 (4): 1-6.

[28] ZHAI Chunxiao, Xie Hui. Study on the correlation between emotional intelligence, empathy ability and nurse-patient communication ability of undergraduate interns [J]. Journal of Nursing Management, 2015, 15 (2): 91-93.

[29] Wang Shengfeng, Qi Yumei. Study on the correlation between emotional intelligence and professional self-concept and job performance of community nurses [J]. Preventive Medicine, 2014, 41 (3): 459-461. 